\title{
Interacting ghost dark energy in complex quintessence theory
}

\author{
Yang Liu \\ Faculty of Physics, Ludwig Maximillian Unisersity Munich, Theresienstrasse 37, 80333 Munich, Germany
}

Received: 7 September 2020 / Accepted: 20 December 2020 / Published online: 28 December 2020

(C) The Author(s) 2020

\begin{abstract}
We employ a ghost model of interacting dark energy to obtain the equation of state $\omega$ for ghost energy density in an FRW universe in complex quintessence theory. We reconstruct the potential and study the dynamics of the scalar field that describes complex quintessence cosmology. We perform $\omega-\omega^{\prime}$ analysis and stability analysis for both non-interacting and interacting cases and find that the same basic conclusion as for the real model, where $\omega^{\prime}=d \omega / d \ln a$. Taking account of the effect of the complex part and assuming the real part of the quintessence field to be a slow-rolling field, we conclude that the non-interacting model cannot describe the real universe since this will lead to fractional energy density $\Omega_{D}>1$, where $\Omega_{D}$ can be defined as the ratio of $\rho_{D}$ to $\rho_{c r}$. However, for the interacting case, if we take present $\Omega_{D}=0.73$, then we can determine that $b^{2}=0.0849$, where $b^{2}$ is the interaction coupling parameter between matter and dark energy. In the real quintessence model, $\Omega_{D}$ and $b^{2}$ are independent parameters, whereas in the complex quintessence model, we conclude that there is a relationship between these two parameters.
\end{abstract}

\section{Contents}

1 Introduction . . . . . . . . . . . 1

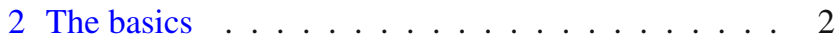

2.1 The complex quintessence field . . . . . . . . . 2

2.2 Ghost dark energy . . . . . . . . . . . . . . 3

3 The complex quintessence field as ghost dark energy in an FRW universe ... . . . . . . . . 3

4 Interacting ghost dark energy in an FRW universe in complex quint-essence theory . . . . . . . . . 5 5

5 Summary and discussion . . . . . . . . . . . . . 7

References .................. . . 8

a e-mail: xijubear2020@Outlook.com (corresponding author)

\section{Introduction}

The accelerated expansion of the universe has been convincingly substantiated by observations on type Ia supernovae [1-3], Large Scale Structure [4,5] and Cosmic Microwave Background anisotropies [6]. In order to explain the reasons for the expansion of the universe, cosmologists have proposed many theoretical models. The most well-accepted explanation at present is dark energy. Dark energy has a negative pressure which leads to an accelerated expansion of universe. However, the nature and cosmological origin of dark energy has not yet been identified.

The most obvious candidate for explaining the nature and origin of dark energy is the cosmological constant $[7,8]$ which has the equation of state $p=-\rho$. However, this explanation itself inevitably leads to further difficulties, such as the fine-tuning problem etc. In view of this, a series of alternative theoretical models have been proposed. In particular, a group of scalar field dark energy theories including quintessence [9-14], K-essence [15,16], tachyon [17-20], phantom [21], ghost condensate [22,23] and quintom [2429], braneworld models [30], interacting dark energy models [31], and Chaplygin gas models [32] etc. have been widely studied.

In addition to the degrees of freedom that already exist in standard cosmological models, most dark energy models introduce even further degrees of freedom. Introducing such further degrees of freedom requires studying their properties and the further consequences arising from them in modeling the universe. In view of this, those new suggestions on the origin of dark energy have been made and which do not require introducing further unknown degrees of freedom whilst yielding the necessary cosmic expansion with a dark energy value of the correct magnitude are of particular interest. Among these models, ghost dark energy (GDE) which uses the so-called Veneziano ghost to account for the observed accelerated expansion of the universe [33-37] is one well-known example. In the GDE model, the cosmolog- 
ical constant is considered to originate from the contribution of ghost fields, which are predicted to exist according to low energy effective theory [38-42]. The ghosts are introduced in order to resolve the $U(1)$ problem [38-42]. It is claimed that the Veneziano ghost field provides important physical spacetime effects within non-topological or dynamic spacetime. The ghost field in curved space leads to a small vacuum energy density which is proportional to $\Lambda_{Q C D}^{3} H$, where $\Lambda^{3}$ is the QCD mass scale and $H$ is the Hubble constant [42-44]. The advantages of this model are that it does not require the introduction of any new parameters or degrees of freedom and that it can be totally embedded in the standard model as well as general relativity. The dynamic behaviour of this model is presented in Ref. [44].

One initial example of scalar-field theory is so-called quintessence [9-14], which is described by a scalar field $Q$ having a slowly decreasing potential $V(Q)$. If the field evolves slowly enough, the kinetic energy density is less than the potential energy density, resulting in negative pressure, which means that the universe will experience an accelerated expansion. Zlatev, Wang and Steinhardt [45] as well as Huterer and Turner [46] have considered the basic properties of real quintessence theory. In most of the subsequent papers, real scalar field cases were considered with only a few papers dealing with complex field cases. Gu and Hwang [47] pointed out the possibility of using a complex scalar field for the quintessence as a way of explaining the acceleration of the universe. Extending the ideas of Huterer and Turner [46] they derived reconstruction equations for the complex field, which demonstrated the feasibility of using complex scalar fields whilst maintaining the uniqueness of the inverse problem. However, Gu and Hwang [43] did not explore the possibility of using a dark energy model in a complex scalar field and more particularly in complex quintessence theory. In this paper, we consider the correspondence between ghost dark energy and the complex quintessence model with the latter being a source of the former.

We first review the basics of complex quintessence field theory as well as ghost dark matter and propose a correspondence between the ghost dark energy scenario and the complex quintessence model. We then reconstruct the potential and study the dynamics of a scalar field that describes complex quintessence cosmology. In Sect. 3, we preform the $\omega-\omega^{\prime}$ analysis and the stability analysis for the noninteracting case, and we find that the basic conclusion is the same as the real model. Taking account of the effect of the complex part and assuming the real part of the quintessence field to be a slow-rolling field, we suggest that a noninteracting model cannot describe the real universe since this will lead to $\Omega_{D}>1$. In Sect. 4, we conduct the $\omega-\omega^{\prime}$ analysis and the stability analysis for the interacting case, which has the same result as for the real model. Considering the contribution of the complex part of quintessence, regarding real part as a slow-rolling field and taking $\Omega_{D}=0.73$, then we can determine that $b^{2}=0.0849$, which is consistent with the observational data [48]. In the real quintessence model, $\Omega_{D}$ and $b^{2}$ are independent parameters, whereas in the complex quintessence model these two parameters are interrelated.

\section{The basics}

\subsection{The complex quintessence field}

We consider the Friedmann-Robertson-Walker (FRW) metric

$d s^{2}=-d t^{2}+a^{2}(t)\left(\frac{d r^{2}}{1-k r^{2}}+r^{2} d \Omega^{2}\right)$

where $k$ is the curvature of space, and $k=0,1,-1$ for a flat, a closed and an open universe respectively. The action of the universe is given by

$S=\int d^{4} x \sqrt{-g}\left(\frac{1}{16 \pi G} R+\rho_{m}+\mathcal{L}_{\Phi}\right)$

where $g$ is the determinant of the metric tensor $g_{\mu \nu}, G$ is the Newtons constant, $R$ is the Ricci scalar, $\rho_{m}$ is the density of ordinary matter, and $\mathcal{L}_{\Phi}$ is the Lagrangian density of the complex quintessence field $\Phi$ given by:

$\mathcal{L}_{\Phi}=-\frac{1}{2} g^{\mu \nu}\left(\partial_{\mu} \Phi^{*}\right)\left(\partial_{\nu} \Phi\right)-V(|\Phi|)$

Here $\mu, v=0,1,2,3$. We have assumed that in Eq. (2.3) that the potential $V$ depends solely on the absolute value of the complex quintessence field: $|\Phi|$ [47].

We can express $\Phi$ in terms of amplitude $\phi$ and phase $\theta$ to as,

$\Phi(x)=\phi(x) e^{i \theta(x)}$

Or more precisely, $\Phi(t)=\phi(t) e^{i \theta(t)}$. We can then rewrite Eq. (2.3) as

$$
\begin{aligned}
\mathcal{L}_{\Phi}= & -\frac{1}{2} g^{\mu \nu}\left(\partial_{\mu} \phi\right)\left(\partial_{\nu} \phi\right) \\
& -\frac{1}{2} \phi^{2} g^{\mu \nu}\left(\partial_{\mu} \theta\right)\left(\partial_{\nu} \theta\right)-V(\phi)
\end{aligned}
$$

By employing the metric of Eq. (2.1), we have the following equations:

$$
\begin{aligned}
H^{2} & \equiv\left(\frac{\dot{a}}{a}\right)^{2}=\frac{8 \pi G}{3} \rho-\frac{k}{a^{2}} \\
& =\frac{8 \pi G}{3}\left\{\rho_{m}+\frac{1}{2}\left(\dot{\phi}^{2}+\phi^{2} \dot{\theta}^{2}\right)+V(\phi)\right\}-\frac{k}{a^{2}} \\
\left(\frac{\ddot{a}}{a}\right)^{2} & =-\frac{4 \pi G}{3}(\rho+3 p) \\
& =-\frac{8 \pi G}{3}\left\{\frac{1}{2} \rho_{m}+\left(\dot{\phi}^{2}+\phi^{2} \dot{\theta}^{2}\right)-V(\phi)\right\}
\end{aligned}
$$




$$
\begin{aligned}
& \ddot{\phi}+3 H \dot{\phi}-\dot{\theta}^{2} \phi+V^{\prime}(\phi)=0 \\
& \ddot{\theta}+\left(2 \frac{\dot{\phi}}{\phi}+3 H\right) \dot{\theta}=0
\end{aligned}
$$

where $H$ is the Hubble parameter, dot and prime represent derivatives with respect to $t$ and $\phi$ respectively, $\rho$ is the energy density where $\rho=\rho_{\Phi}+\rho_{m}$, and $p$ is the pressure. Equations (2.6) and (2.9) are the fundamental equations which govern the evolution of the universe. Equations (2.6) and (2.7) are Friedmann equations for this model. From Eq. (2.5) we can derive the energy density $\rho_{\Phi}$ and pressure $p_{\Phi}$ to be:

$\rho_{\Phi}=\frac{1}{2}\left(\dot{\phi}^{2}+\phi^{2} \dot{\theta}^{2}\right)+V(\phi)$

$p_{\Phi}=\frac{1}{2}\left(\dot{\phi}^{2}+\phi^{2} \dot{\theta}^{2}\right)-V(\phi)$

Equation (2.9) can be solved to yield the angular velocity, i.e. the time derivative of $\theta$ which is given by

$\dot{\theta}=\frac{\omega}{a^{3} \phi^{2}}$

where $\omega$ is an integration constant which is determined by the value of $\dot{\theta}$ initially or at some specific time. By using Eq. (2.12), Eqs. (2.6)-(2.9) can be rewritten in terms of $\phi$, as presented in detail in Ref. [47] to which reference is made.

\subsection{Ghost dark energy}

Following Ref. [48], let us breifly review the ghost dark energy model briefly. For a non-flat FRW universe filled with dark energy and dust (dark matter), the corresponding Friedmann equation is written as

$H^{2}+\frac{k}{a^{2}}=\frac{8 \pi G}{3}\left(\rho_{m}+\rho_{D}\right)$

where $\rho_{D}$ and $\rho_{m}$ are the energy densities of dark energy and pressureless matter respectively.

The ghost energy density in standard cosmology is defined by

$\rho_{D}=\alpha H$

where $\alpha$ denotes a constant of order $\Lambda_{Q C D}^{3}$ and $\Lambda_{Q C D}$ represents the QCD mass scale and $H$ is the Hubble constant. Here $\Lambda_{Q C D}$ is approximately equal to $100 \mathrm{MeV}$ and $H$ is approximately equal to $10^{-33} \mathrm{eV}$, so $\Lambda_{Q C D}^{3} H$ gives the correct order of magnitude $\left(3 \times 10^{-3} \mathrm{eV}\right)^{4}$ for the presently observed dark energy value. This numerical coincidence is quite remarkable and also means that this model can obviate the fine-tuning problem [33-37].

We define the curvature energy density $\rho_{k}$ and the critical energy density $\rho_{c r}$ as usual as:

$$
\begin{aligned}
\rho_{k} & =\frac{3 k}{8 \pi G a^{2}} \\
\rho_{c r} & =3 M_{p}^{2} H^{2}
\end{aligned}
$$

We also introduce three fractional energy densities $\Omega_{D}, \Omega_{m}$ and $\Omega_{k}$ :

$$
\begin{aligned}
& \Omega_{D}=\frac{\rho_{D}}{\rho_{c r}}=\frac{\alpha}{3 M_{p}^{2} H} \\
& \Omega_{m}=\frac{\rho_{m}}{\rho_{c r}}=\frac{\rho_{m}}{3 M_{p}^{2} H^{2}} \\
& \Omega_{k}=\frac{\rho_{k}}{\rho_{c r}}=\frac{k}{H^{2} a^{2}}
\end{aligned}
$$

Then the Friedmann equation can then be written as:

$$
1+\Omega_{k}=\Omega_{D}+\Omega_{m}
$$

If there is no interaction between matter and ghost dark energy, i.e.,

$$
\begin{aligned}
& \dot{\rho}_{D}+3 H \rho_{D}\left(1+\omega_{D}\right)=0 \\
& \dot{\rho}_{m}+3 H \rho_{m}=0
\end{aligned}
$$

then for the ghost dark energy density the equation of state $[48,49]$ can be derived to be:

$\omega_{D}=-\frac{1}{2-\Omega_{D}}\left(1-\frac{\Omega_{k}}{3}\right)$

If there is an interaction between matter and ghost dark energy, i.e.,

$$
\begin{aligned}
& \dot{\rho}_{D}+3 H \rho_{D}\left(1+\omega_{D}\right)=-Q \\
& \dot{\rho}_{m}+3 H \rho_{m}=Q
\end{aligned}
$$

where $Q$ is the interaction term, then we define $Q$ as

$Q=3 b^{2} H\left(\rho_{m}+\rho_{D}\right)=3 b^{2} H \rho_{D}(1+u)$

with $b^{2}$ a coupling parameter. Here $u$ is defined as

$u=\frac{\rho_{m}}{\rho_{D}}=\frac{\Omega_{m}}{\Omega_{D}}=\frac{1-\Omega_{D}}{\Omega_{D}}$

Under these assumptions for the ghost dark energy density, the equation of state $[48,49]$ can be derived to be:

$\omega_{D}=-\frac{1}{2-\Omega_{D}}\left(1-\frac{\Omega_{k}}{3}+\frac{2 b^{2}}{\Omega_{D}}\left(1+\Omega_{k}\right)\right)$

Further details of ghost dark energy can be found in Ref. $[48,49]$ to which reference is made.

\section{The complex quintessence field as ghost dark energy in an FRW universe}

Considering first the case without interaction and establishing the correspondence between the energy density of the complex quintessence field and ghost dark energy, then using Eqs. (2.10), (2.12) and (2.14), we obtain

$\rho_{D}=\frac{1}{2}\left(\dot{\phi}^{2}+\frac{\omega^{2}}{a^{6} \phi^{2}}\right)+V(\phi)=\alpha H$ 
For brevity, if we set

$A=\frac{1}{2}\left(\dot{\phi}^{2}+\frac{\omega^{2}}{a^{6} \phi^{2}}\right)$

then we have

$V(\phi)=\alpha H-A$

On the other hand, if we establish the correspondence between the energy density of the complex quintessence field and the ghost dark energy, then we have

$\omega_{\Phi} \equiv \frac{p_{\Phi}}{\rho_{\Phi}}=\omega_{D}$

Combining Eqs. (2.10), (2.11) and (2.23), we obtain

$\frac{A-V(\phi)}{A+V(\phi)}=-\frac{1}{2-\Omega_{D}}\left(1-\frac{\Omega_{k}}{3}\right)$

Rewriting the potential $V(\phi)$ gives

$V(\phi)=\frac{3-\Omega_{D}-\frac{\Omega_{k}}{3}}{1-\Omega_{D}+\frac{\Omega_{k}}{3}} A$

Combining Eqs. (3.3) and (3.6), gives

$\frac{3-\Omega_{D}-\frac{\Omega_{k}}{3}}{1-\Omega_{D}+\frac{\Omega_{k}}{3}} A=\alpha H-A$

so that, then we have

$H=\frac{1}{\alpha}\left(\frac{4-2 \Omega_{D}}{1-\Omega_{D}+\frac{\Omega_{k}}{3}}\right) A$

In this article, we principally consider the case for $k=0$. We will conduct $\omega-\omega^{\prime}$ analysis, stability analysis and further physical interpretation for the non-interacting model.

The $\omega-\omega^{\prime}$ analysis is an important tool to discriminate different models as was first proposed in Ref. [50] and subsequently extended in Refs. [51,52]. Here $\omega$ is the equationof-state parameter $\omega \equiv p / \rho$, while $\omega^{\prime}$ is the derivative of $\omega$ with respect to lna. Based on Ref. [48], in flat space $k=0$, so we have

$\frac{d \Omega_{D}}{d \ln a}=3 \Omega_{D} \frac{1-\Omega_{D}}{2-\Omega_{D}}$

and

$\omega_{D}=-\frac{1}{2-\Omega_{D}}$

So that we obtain

$\frac{d \omega_{D}}{d \ln a}=-\frac{3 \Omega_{D}\left(1-\Omega_{D}\right)}{\left(2-\Omega_{D}\right)^{3}}$

Based on Eqs. (3.10), (3.11) can be rewritten as

$\frac{d \omega_{D}}{d \ln a}=-3 \omega_{D}^{3}\left(2+1 / \omega_{D}\right)\left(1+1 / \omega_{D}\right)$

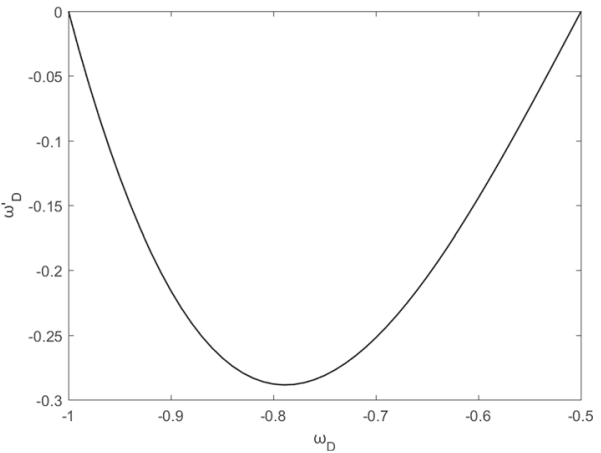

Fig. 1 Evolution trajectories of $\omega_{D}-\omega_{D}^{\prime}$ for the non-interacting case

where $-1<\omega_{D}<-\frac{1}{2}$. From Eq. (3.12), we know that $\frac{d \omega_{D}}{d \ln a}$ is always negative, namely, $\omega$ decreases monotonically with the increase of $\ln a$. Figure 1 shows the evolution trajectories of $\omega-\omega^{\prime}$. From Fig. 1, we know that when $\omega_{D}$ is about 0.8 , $\omega_{D}^{\prime}$ has a minimum. In addition, when $\omega_{D}$ equals to -1 or $-0.5, \omega_{D}^{\prime}$ equals to 0 .

We can also determine the stability of this model by defining the velocity of sound according to,

$v_{s}^{2}(z) \equiv d p_{D} / d \rho_{D}=\frac{d p_{D} / d z}{d \rho_{D} / d z}$

where $1+z=a^{-1}$ and $z$ is the redshift. In flat space, $k=0$, the Hubble constant $H$ can be written as [48]

$H=\frac{\alpha(1+u)}{3 M_{p}^{2}}$

where $M_{p}^{2}=1 / 8 \pi G$ and $u=\rho_{m} / \rho_{D}$. We can then obtain,

$\frac{d H}{d a}=-\frac{\alpha}{M_{p}^{2} \Omega_{D} a} \frac{1-\Omega_{D}}{2-\Omega_{D}}$

Then we have

$\frac{d \rho_{D}}{d z}=\alpha \frac{d H}{d z}=\alpha\left(-a^{2}\right) \frac{d H}{d a}=\frac{\alpha^{2} a}{M_{p}^{2} \Omega_{D}} \frac{1-\Omega_{D}}{2-\Omega_{D}}>0$

The stability of this model is therefore determined by the sign of $d p_{D} / d z$. Since $p_{D}=\omega_{D} \rho_{D}$, then

$\frac{d p_{D}}{d z}=\frac{d \omega_{D}}{d z} \rho_{D}+\omega \frac{d \rho_{D}}{d z}=-a \frac{d \omega_{D}}{d \ln a} \rho_{D}+\omega \frac{d \rho_{D}}{d z}$

Combining Eqs. (3.11), (3.13), (3.16) and (3.17), we can then derive that

$v_{s}^{2} \equiv d p_{D} / d \rho_{D}=\frac{2 \Omega_{D}-2}{\left(2-\Omega_{D}\right)^{2}}$

If we require this model to be stable, then $v_{s}^{2}(z)$ must be larger than zero. Recalling Eq. (3.18) this means that $\Omega_{D}>1$ which is impossible. Figure 2 shows the evolution of $v_{s}^{2}$ against $\Omega_{D}$ for the non-interacting case. Since the magnitude of the speed of sound cannot be negative, a non-interacting ghost dark 


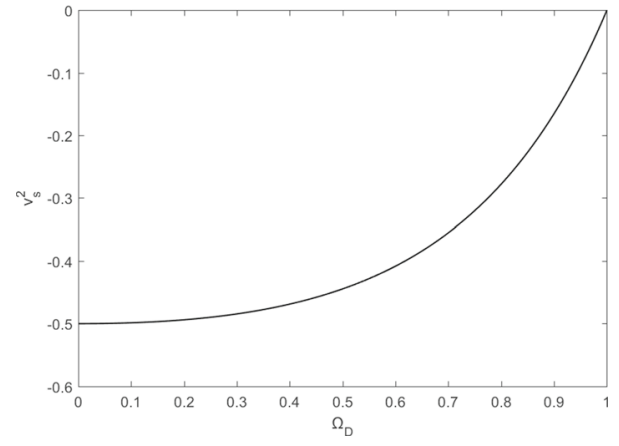

Fig. 2 Evolution of the speed of sound squared $v_{s}^{2}$ versus $\Omega_{D}$ for the non-interacting case

energy dominated universe in the future cannot be expected to be the fate of the universe. In fact, this is a general conclusion for ghost dark energy model irrespective of whether the complex part of scalar field is considered or not. Similar results are presented in Ref. [49] to which reference is made.

We can expect that the above $\omega-\omega^{\prime}$ analysis and stability analysis for the complex quintessence model to yield the same results when using the real quintessence model, since the two analyses only involve the ghost dark energy model which is entirely unrelated to any effect from the complex part. We will now consider the effect of the complex part of the quintessence field. In this article, we restrict our considerations to the effect of a slow-rolling field.

Combining Eqs. (2.10), (2.11) and (3.2), we can derive,

$\alpha H \frac{1-\Omega_{D}}{2-\Omega_{D}}=\dot{\phi}^{2}+\frac{\omega^{2}}{a^{6} \phi^{2}}$

Considering only a slow-rolling field, i.e., ignoring the term $\dot{\phi}^{2}$, we have

$\alpha H \frac{1-\Omega_{D}}{2-\Omega_{D}}=\frac{\omega^{2}}{a^{6} \phi^{2}}$

So that

$\phi=\frac{\omega}{a^{3}} \sqrt{\frac{2-\Omega_{D}}{\left(1-\Omega_{D}\right) \alpha H}}$

We consider only the positive solution. Since $\dot{\phi}=H \frac{d \phi}{d \ln a}$ and $H$ cannot be ignored, then $\frac{d \phi}{d \ln a} \approx 0$. Combining Eqs. (3.9), (3.15) and (3.21), we have

$$
\begin{aligned}
\frac{d \phi}{d \ln a}= & \frac{-3 \omega}{a^{3}} \sqrt{\frac{2-\Omega_{D}}{\left(1-\Omega_{D}\right) \alpha H}} \\
& +\frac{\omega}{2 a^{3}} \frac{1}{\sqrt{\frac{2-\Omega_{D}}{\left(1-\Omega_{D}\right) \alpha H}}} \frac{3\left(\Omega_{D}^{2}-2 \Omega_{D}+2\right)}{\alpha H\left(\Omega_{D}^{2}-3 \Omega_{D}+2\right)} \approx 0
\end{aligned}
$$

After rearranging terms, we obtain,

$\Omega_{D}^{2}-6 \Omega_{D}+6=0$

Whose solutions are $\Omega_{D}=3 \pm \sqrt{3}$, which is inconsistent with the assumption $\Omega_{D}<1$. We can therefore conclude that, if we regard the complex quintessence field as ghost dark energy and make the slow-rolling field assumption, the noninteracting case cannot be used to describe the real universe. We therefore need to consider the case of interaction between matter and ghost dark energy. In the next section, we will determine the value of $b^{2}$ in Eq. (2.26).

\section{Interacting ghost dark energy in an FRW universe in complex quint-essence theory}

We now consider the case of interaction between matter and ghost dark energy. Combining Eqs. (2.17), (2.19) and (2.28), we obtain

$$
\begin{aligned}
\omega_{D}= & -\frac{1}{2-\frac{\alpha}{3 M_{p}^{2} H}} \\
& \times\left\{1-\frac{k}{3 H^{2} a^{2}}+\frac{6 b^{2} M_{p}^{2} H}{\alpha}\left(1+\frac{k}{H^{2} a^{2}}\right)\right\}
\end{aligned}
$$

In particular, for a flat universe, $k=0$ and $\Omega_{k}=0$, then

$$
\begin{aligned}
\omega_{D} & =-\frac{1}{2-\Omega_{D}}\left(1+\frac{2 b^{2}}{\Omega_{D}}\right) \\
& =-\frac{1}{2-\frac{\alpha}{3 M_{p}^{2} H}}\left(1+\frac{6 b^{2} M_{p}^{2} H}{\alpha}\right)
\end{aligned}
$$

For brevity, we set

$$
\begin{aligned}
B= & -\omega_{D}=\frac{1}{2-\frac{\alpha}{3 M_{p}^{2} H}} \\
& \times\left\{1-\frac{k}{3 H^{2} a^{2}}+\frac{6 b^{2} M_{p}^{2} H}{\alpha}\left(1+\frac{k}{H^{2} a^{2}}\right)\right\}
\end{aligned}
$$

If we establish the correspondence between the energy density of the complex quintessence field and the ghost dark energy, then we obtain,

$\omega_{D}=\omega_{\Phi}$

that is to say,

$$
-B=\frac{A-V(\phi)}{A+V(\phi)}
$$

so that we can obtain

$$
V(\phi)=-\frac{B+1}{B-1} A
$$


Combining Eqs. (3.3) and (4.6), we have

$-\frac{B+1}{B-1} A=\alpha H-A$

therefore

$H=-\frac{2}{\alpha} \frac{1}{B-1} A=\frac{2}{\alpha} \frac{1}{\omega_{D}+1} A$

In order to be self-consistent, $B$ must be smaller than 1, i.e.,

$\frac{1}{2-\Omega_{D}}\left\{1-\frac{k}{3 H^{2} a^{2}}+\frac{2 b^{2}}{\Omega_{D}}\left(1+\frac{k}{H^{2} a^{2}}\right)\right\}<1$

To ensure the required accelerated expansion of the universe, based on Eq. (2.7) we have,

$\rho_{m}<2\left(V(\phi)-\left(\dot{\phi}^{2}+\phi^{2} \dot{\theta}^{2}\right)\right)=2 V(\phi)-4 A$

Considering Eq. (3.3), we have,

$\rho_{m}<2 V(\phi)-4 A=6 V(\phi)-4 \alpha H$

therefore

$V(\phi)>\frac{2}{3} \alpha H$

Combining Eqs. (3.3) and (4.12), we have as the constraint for $V(\phi)$,

$\frac{2}{3} \alpha H<V(\phi)<\alpha H$

We will now perform the $\omega-\omega^{\prime}$ analysis for interacting case in flat space $k=0$. Considering Eq. (2.28), we have

$\omega_{D}=-\frac{1}{2-\Omega_{D}}\left(1+\frac{2 b^{2}}{\Omega_{D}}\right)$

So that

$\omega_{D}^{\prime}=\frac{\frac{2 b^{2}}{\Omega_{D}^{2}} \Omega_{D}^{\prime}\left(2-\Omega_{D}\right)-\Omega_{D}^{\prime}\left(1+\frac{2 b^{2}}{\Omega_{D}}\right)}{\left(2-\Omega_{D}\right)^{2}}$

where ' denotes the derivative with respect to lna. Based on Ref. [48], we have

$\frac{d \Omega_{D}}{d \ln a}=\frac{3}{2}\left[1-\frac{\Omega_{D}}{2-\Omega_{D}}\left(1+\frac{2 b^{2}}{\Omega_{D}}\right)\right]$

Therefore,

$\omega_{D}^{\prime}=\frac{\left[1-\frac{\Omega_{D}}{2-\Omega_{D}}\left(1+\frac{2 b^{2}}{\Omega_{D}}\right)\right]\left(\left(\frac{6 b^{2}}{\Omega_{D}}-\frac{3}{2} \Omega_{D}-6 b^{2}\right)\right.}{\left(2-\Omega_{D}\right)^{2}}$

In particular, when $b^{2}=0$, Eq. (4.13) becomes Eq. (3.12). Figure 3 shows the evolution of $\omega-\omega^{\prime}$ for different values of $b^{2}$. Here we have taken $\Omega_{D}=0.73$ [48]. From Fig. 3, we conclude that for the interacting case, there exists a overlapping region in which a single value of $\omega_{D}$ corresponds to two possible values of $\omega_{D}^{\prime}$. In particular, the width of the

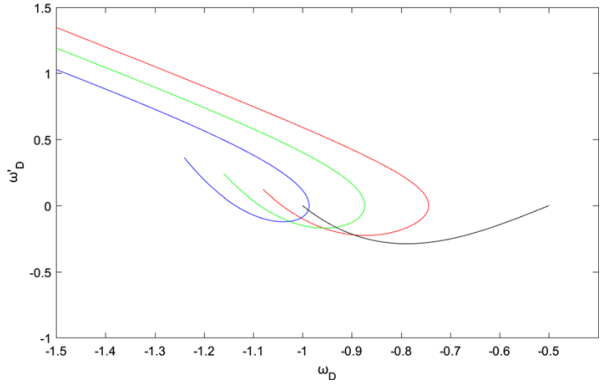

Fig. 3 Evolution trajectories of $\omega_{D}-\omega_{D}^{\prime}$ for different values of $b^{2}$. The red line corresponds to $b^{2}=0.04$, the green line to $b^{2}=0.08$, the blue line to $b^{2}=0.12$ and the black line to $b^{2}=0$

region becomes narrower with the increasing of $b^{2}$. In contrast to this, for the non-interacting case, there is no overlapping region at all. This result is different from that in an agegraphic dark energy model [53]. In fact, the $\omega-\omega^{\prime}$ analysis is a useful dynamic analysis for discriminating different dark energy models [53].

Continuing with the above, we can also determine the stability of this model for the interacting case. Using a similar calculation, we obtain,

$$
\begin{aligned}
\frac{d H}{d a} & =-\frac{\alpha}{2 a \Omega_{D} M_{p}^{2}}\left[1-\frac{\Omega_{D}}{2-\Omega_{D}}\left(1+\frac{2 b^{2}}{\Omega_{D}}\right)\right] \\
\frac{d \rho_{D}}{d a} & =\frac{\alpha^{2} a}{2 \Omega_{D} M_{p}^{2}}\left[1-\frac{\Omega_{D}}{2-\Omega_{D}}\left(1+\frac{2 b^{2}}{\Omega_{D}}\right)\right]
\end{aligned}
$$

and

$$
\begin{aligned}
\frac{d p_{D}}{d a}= & -\frac{1}{\left(2-\Omega_{D}\right)^{2}} \frac{3}{2} \Omega_{D} a \rho_{D}\left(\frac{4 b^{2}}{\Omega_{D}^{2}}-\frac{4 b^{2}}{\Omega_{D}}-1\right) \\
& \times\left[1-\frac{\Omega_{D}}{2-\Omega_{D}}\left(1+\frac{2 b^{2}}{\Omega_{D}}\right)\right] \\
& -\frac{1}{2-\Omega_{D}}\left(1+\frac{2 b^{2}}{\Omega_{D}}\right) \frac{\alpha^{2} a}{2 \Omega_{D} M_{p}^{2}} \\
& \times\left[1-\frac{\Omega_{D}}{2-\Omega_{D}}\left(1+\frac{2 b^{2}}{\Omega_{D}}\right)\right]
\end{aligned}
$$

The square of the velocity of sound can then be obtained as,

$v_{s}^{2} \equiv d p_{D} / d \rho_{D}=\frac{2 \Omega_{D}^{2}-2 \Omega_{D}+6 b^{2} \Omega_{D}-8 b^{2}}{\Omega_{D}\left(2-\Omega_{D}\right)^{2}}$

For the model to be stable, then $v_{s}^{2}$ must be larger than zero. This means that $\Omega_{D}$ is larger than $\frac{4}{3}$, which is impossible. We can therefore conclude that for the interacting case, the model is still unstable and cannot conclude that a ghost dark energy dominated universe will be the future is the fate of the real universe. Figure 4 shows the evolution of $v_{s}^{2}$ against $\Omega_{D}$ for different $b^{2}$. Further details of the stability analysis can be found in Ref. [49]. 


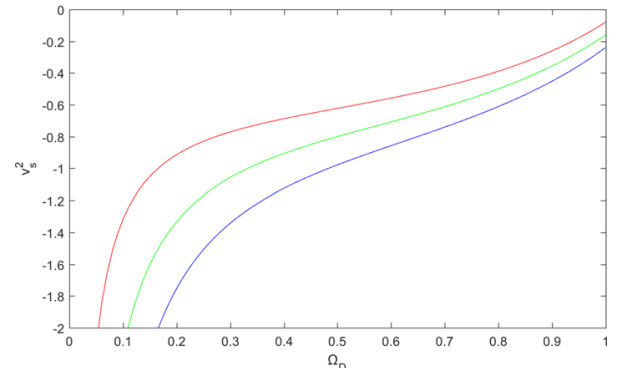

Fig. 4 Evolution of the speed of sound squared $v_{s}^{2}$ versus $\Omega_{D}$ for different values of $b^{2}$. The red line corresponds to $b^{2}=0.04$, the green line to $b^{2}=0.08$ and the blue line to $b^{2}=0.12$

The above $\omega-\omega^{\prime}$ analysis and stability analysis yield the same results with a real quintessence scalar field, since the two analyses only involve the ghost dark energy model and do not include the effect of the complex part of quintessence. We will now consider the effect of the complex part of the quintessence field for the interacting case. As mentioned above, we only consider the effect of a slow-rolling field.

As for the interacting case in flat space, we can then obtain

$\alpha H \frac{\Omega_{D}\left(1-\Omega_{D}\right)-2 b^{2}}{\Omega_{D}\left(2-\Omega_{D}\right)} \approx \frac{\omega^{2}}{a^{6} \phi^{2}}$

Therefore, we have

$\phi=\frac{\omega}{a^{3}} \sqrt{\frac{\Omega_{D}\left(2-\Omega_{D}\right)}{\Omega_{D}\left(1-\Omega_{D}\right)-2 b^{2}} \frac{1}{\alpha H}}$

If we require $\frac{d \phi}{d l n a} \approx 0$, then we obtain

$$
\begin{aligned}
0 \approx & -6 \Omega_{D}\left(2-\Omega_{D}\right)\left[\Omega_{D}\left(1-\Omega_{D}\right)-2 b^{2}\right] \\
& +\left[3-\frac{3 \Omega_{D}}{2-\Omega_{D}}\left(1+\frac{2 b^{2}}{\Omega_{D}}\right)\right. \\
& \left.-3 \Omega_{D}+\frac{3 \Omega_{D}^{2}}{2-\Omega_{D}}\left(1+\frac{2 b^{2}}{\Omega_{D}}\right)\right] \\
& \times\left[\Omega_{D}\left(1-\Omega_{D}\right)-2 b^{2}\right] \Omega_{D} \\
& -\left(2-\Omega_{D}\right)\left[\frac{3}{2} \Omega_{D}-\frac{3}{2} \frac{\Omega_{D}^{2}}{2-\Omega_{D}}\left(1+\frac{2 b^{2}}{\Omega_{D}}\right)\right. \\
& \left.-3 \Omega_{D}^{2}+\frac{3 \Omega_{D}^{3}}{2-\Omega_{D}}\left(1+\frac{2 b^{2}}{\Omega_{D}}\right)\right] \\
& +\frac{3}{2} \Omega_{D}\left[\Omega_{D}\left(1-\Omega_{D}\right)-2 b^{2}\right] \\
& \times\left[2-\Omega_{D}-\Omega_{D}\left(1+\frac{2 b^{2}}{\Omega_{D}}\right)\right]
\end{aligned}
$$

If we take the present $\Omega_{D}=0.73$ [48] and insert this value in Eq. (4.24), we have

$6.2424 b^{4}+8.0893 b^{2}-0.7317=0$ from which it follows that the only reasonable solution for $b^{2}$, is $b^{2}=0.0849$. This numerical result for $b^{2}$ is consistent with the observational result of $b^{2}$ [48]. In Ref. [48], the observational result is $b^{2}=0.09$. However, in the previous research, people only considered the real part of quintessence field so that $b^{2}$ was a free parameter in this model and could only be determined by observation. In this article, we consider the effect of the complex part of the quintessence field. We find that the non-interacting case cannot describe the real universe and an interaction between matter and dark energy must be added. In fact, Eq. (4.24) defines the relationship between $\Omega_{D}$ and $b^{2}$. Therefore, $\Omega_{D}$ cannot be an arbitrary value between 0 and 1 . Its value must instead ensure the existence of a positive solution of Eq. (4.24) for $b^{2}$.

\section{Summary and discussion}

To address the problem of accounting for the accelerated expansion of the universe and due to an absence of knowledge in this domain, theoretical cosmologists have considered a variety of dark matter candidates to explain this phenomenon. The ghost dark matter (GDE) model which was proposed by Urban and Zhitnitsky [33-36] as well as N. Ohta [37] has been studied in several papers $[44,48,49]$. Without introducing further unknown degrees of freedom, this model predicts cosmic expansion based on dark energy with a correct magnitude.

Most previous papers focused on the effects of dark energy using different real scalar field theories, such as quintessence [9-14], K-essence [15,16], tachyon [17-20] etc. However, only a few studies have investigated the possibility of using an approach based on complex scalar fields. Gu and Hwang [47] pointed out the possibility of using complex scalar fields for quintessence as a way of explaining the acceleration of the universe. Complex scalar fields are already known to play an important role in elementary particle physics such as in the mass generation mechanism (Higgs mechanism). The physics of complex fields therefore deserves attention as a way of constructing practical models of our universe.

In this paper, we have used the ghost model of interacting dark energy to obtain the equation of state for the ghost energy density in an FRW universe in complex quintessence theory. We have derived the potential and studied the dynamics of the scalar field that describe complex quintessence cosmology. In Sect. 3, we performed the $\omega-\omega^{\prime}$ analysis and the stability analysis for the non-interacting case and found that the basic conclusion is the same as for the real model. Taking into account the effect of the complex part and regarding the real part of the quintessence field as a slow-rolling field, we concluded that the non-interacting model cannot describe the real universe since this will lead to $\Omega_{D}>1$. In Sect. 4, we performed the $\omega-\omega^{\prime}$ analysis and the stability analysis for 
the interacting case, which lead to the same result as for the real model. Considering the contribution of the complex part of quintessence, regarding the real part as a slow-rolling field and taking the value of $\Omega_{D}=0.73$ we determined the value of $b^{2}=0.0849$, which is consistent with the observational data value $b^{2}=0.09$. [48] In the real model, $\Omega_{D}$ and $b^{2}$ are independent parameters, whereas in the complex model, we found an interrelationship between these two parameters.

Future work can be directed along at least four lines of further research. Firstly, we can establish the correspondence between the complex quintessence field and other dark energy scenarios. In fact, since the ghost dark energy model is unstable, a future universe dominated by ghost dark energy cannot be the fate of the real universe. In subsequent work, we intend to correlate the complex quintessence model and the holographic dark energy model as well as compare these results with the ghost dark energy model. Secondly, we can generalize real scalar-tensor field theories, such as the Brans-Dicke theory to complex versions and then consider dark energy models in generalized scalar-tensor field theory. Thirdly, we can combine complex scalar field theory with elementary particle physics theory to help us build practical models of the universe. Finally, we need to consider how to ensure compatibility of these results with fundamental theories, such as string theory and loop quantum gravity. There is therefore great potential for development of this work in the future.

Data Availability Statement This manuscript has no associated data or the data will not be deposited. [Authors' comment: No data has been used for this article.]

Open Access This article is licensed under a Creative Commons Attribution 4.0 International License, which permits use, sharing, adaptation, distribution and reproduction in any medium or format, as long as you give appropriate credit to the original author(s) and the source, provide a link to the Creative Commons licence, and indicate if changes were made. The images or other third party material in this article are included in the article's Creative Commons licence, unless indicated otherwise in a credit line to the material. If material is not included in the article's Creative Commons licence and your intended use is not permitted by statutory regulation or exceeds the permitted use, you will need to obtain permission directly from the copyright holder. To view a copy of this licence, visit http://creativecomm ons.org/licenses/by/4.0/.

Funded by SCOAP ${ }^{3}$.

\section{References}

1. A.G. Riess et al., Astron. J. 116, 1009 (1998)

2. S. Perlmutter et al., Astron. J. 517, 565 (1999)

3. P. Astier et al., Astron. Astrophys. 447, 31 (2006)

4. K. Abazajian et al., Astron. J. 128, 502 (2004)
5. K. Abazajian et al., Astron. J. 129, 1755 (2005)

6. D.N. Spergel et al., Astrophys. J. Suppl. 148, 175 (2003)

7. V. Sahni, A.A. Starobinsky, Int. J. Mod. Phys. D 9, 373 (2000)

8. P.J.E. Peebles, B. Ratra, Rev. Mod. Phys. 75, 559 (2003)

9. P.J.E. Peebles, B. Ratra, Astrophys. J. 325, L17 (1988)

10. B. Ratra, P.J.E. Peebles, Phys. Rev. D 37, 3406 (1988)

11. C. Wetterich, Nucl. Phys. B 302, 668 (1988)

12. J.A. Frieman, C.T. Hill, A. Stebbins, I. Waga, Phys. Rev. Lett. 75, 2077 (1995)

13. M.S. Turner, M.J. White, Phys. Rev. D 56, 4439 (1997)

14. R.R. Caldwell, R. Dave, P.J. Steinhardt, Phys. Rev. Lett. 80, 1582 (1998)

15. C. Armendariz-Picon, V.F. Mukhanov, P.J. Steinhardt, Phys. Rev. Lett. 85, 4438 (2000)

16. C. Armendariz-Picon, V.F. Mukhanov, P.J. Steinhardt, Phys. Rev. D 63, 103510 (2001)

17. R.R. Caldwell, Phys. Lett. B 545, 23 (2002)

18. R.R. Caldwell, M. Kamionkowski, N.N. Weinberg, Phys. Rev. Lett. 91, 071301 (2003)

19. R.S. Nojiri, S.D. Odintsov, Phys. Lett. B 562, 147 (2003)

20. R.S. Nojiri, S.D. Odintsov, Phys. Lett. B 565, 1 (2003)

21. A. Sen, JHEP 0207, 065 (2002)

22. N. Arkani-Hamed, H.C. Cheng, M.A. Luty, S. Mukohyama, JHEP 0405, 074 (2004)

23. F. Piazza, S. Tsujikawa, JCAP 0407, 004 (2004)

24. B. Feng, X.L. Wang, X.M. Zhang, Phys. Lett. B 607, 35 (2005)

25. Z.K. Guo, Y.S. Piao, X.M. Zhang, Y.Z. Zhang, Phys. Lett. B 608, 177 (2005)

26. X. Zhang, Commun. Theor. Phys. 44, 762 (2005)

27. A. Anisimov, E. Babichev, A. Vikman, JCAP 0506, 006 (2005)

28. E. Elizalde, S. Nojiri, S.D. Odintsov, Phys. Rev. D 70, 043539 (2004)

29. S. Nojiri, S.D. Odintsov, S. Tsujikawa, Phys. Rev. D 71, 063004 (2005)

30. C. Deffayet, G.R. Dvali, G. Gabadadze, Phys. Rev. D 65, 044023 (2002)

31. L. Amendola, Phys. Rev. D 62, 043511 (2000)

32. A.Y. Kamenshchik, U. Moschella, V. Pasquier, Phys. Lett. B 511, $265(2001)$

33. F.R. Urban, A.R. Zhitnitsky, Phys. Lett. B 9, 688 (2010)

34. F.R. Urban, A.R. Zhitnitsky, Phys. Rev. D 063001, 80 (2009)

35. F.R. Urban, A.R. Zhitnitsky, JCAP 018, 0909 (2009)

36. F.R. Urban, A.R. Zhitnitsky, Nucl. Phys. B 135, 835 (2010)

37. N. Ohta, Phys. Lett. B 41, 695 (2011)

38. E. Witten, Nucl. Phys. B 269, 156 (1979)

39. G. Veneziano, Nucl. Phys. B 213, 159 (1979)

40. C. Rosenzweig, J. Schechter, C.G. Trahern, Phys. Rev. D 3388, 21 (1980)

41. P. Nath, R.L. Arnowitt, Phys. Rev. D 473, 23 (1981)

42. K. Kawarabayashi, N. Ohta, Nucl. Phys. B 477, 175 (1980)

43. N. Ohta, Prog. Theor. Phys. 1408, 66 (1981)

44. R.G. Cai, Z.L. Tuo, H.B. Zhang, arXiv:1011.3212

45. I. Zlatev, L. Wang, P.J. Steinhardt, Phys. Rev. Lett. 82, 896 (1999)

46. D. Huterer, M.S. Turner, Phys. Rev. D 60, 081301 (1999)

47. J. Gu, W. Hwang, Phys. Lett. B 517, 1 (2001)

48. A. Sheykhi, M. Sadegh Movahed, Gen. Rel. Grav 44, 449 (2012)

49. E. Ebrahimi, A. Sheykhi, arXiv: 1105.5680 [hep-th]

50. R.R. Caldwell, E.V. Linder, Phys. Rev. Lett. 95, 141301 (2005)

51. T. Chiba, Phys. Rev. D 73, 063501 (2006)

52. R.J. Scherrer, Phys. Rev. D 73, 043502 (2006)

53. H. Wei, R.-G. Cai, Phys. Lett. B 655, 1-6 (2007) 